\title{
PROCESSOS DE CUSTOMIZAÇÃO DE ÓRTESES: ETAPAS, TECNOLOGIAS E MEIO DE FABRICAÇÃO UTILIZADOS.
}

\author{
ORTHESIS CUSTOMIZATION PROCESSES: STAGES, TECHNOLOGIES AND \\ MEANS OF MANUFACTURE USED.
}

Ricardo Schwinn Rodrigues, Bel. ${ }^{1}$, Márcia Cattoi Schmidt, Bel. ${ }^{2}$, Diogo Gonçalves Martins, Bel. ${ }^{3}$

(1)Universidade do Estado de Santa Catarina

e-mail: rsr404@ hotmail.com

(2)Universidade do Estado de Santa Catarina

e-mail: mcattoi@hotmail.com

(3)Universidade do Estado de Santa Catarina

e-mail: diogo3martins@gmail.com

Palavras chave: processo de customização, órtese, design.

\begin{abstract}
A atenção aos aspectos ergonômicos no desenvolvimento das órteses customizadas permite a adequação destes dispositivos à geometria das sessões do corpo de um indivíduo específico, às debilidades que se pretende corrigir e às tarefas a serem realizadas com apoio do dispositivo. Nesse contexto, o objetivo deste estudo foi o de identificar processos autorais de especialistas para o desenvolvimento e fabricação de órteses customizadas, identificando as etapas, tecnologias e os meios de fabricação utilizados. Para tal, realizou-se uma revisão bibliográfica sistematizada, na qual foram selecionados 05 estudos que apresentam processos próprios de desenvolvimento de órteses customizadas. Foram detectados os seguintes pontos focais: 1. A automatização do processo 2. A utilização de novas tecnologias não invasivas na obtenção de dados antropométricos (como scanners 3D e Tomografia Computadorizada) que permitem liberdade de criação em softwares CAD. 3. A tarefa a ser realizada. 4. Redução das etapas e do tempo consumido nas mesmas.
\end{abstract}

Keywords: customization process, orthosis, design.

The attention to the ergonomic aspects in the development of customized orthoses allows the adaptation of these devices to the body session's geometry of a specific individual, to the deficiencies intended to correct and the tasks to be carried out with support of the device. In this context, the objective of this study was to identify author's processes from specialists for development and manufacture of customized orthoses, identifying the stages, technologies and the means of manufacture used. Therefore, a systematic bibliographic review was carried out, in which five studies were selected presenting its own processes of development of customized orthoses. The following focal points were detected: 1. Process automation 2. The use of new non-invasive technologies to obtain anthropometric data (such as $3 D$ scanners and CT scanners) allows freedom of creation in CAD software. 3. Task to be performed. 4. Reduction of the steps and the time consumed in them. 


\section{$16^{\circ}$ \\ ERGODESIGN USIHC CINAHPA}

$16^{\circ}$ Ergodesign - Congresso Internacional de Ergonomia e Usabilidade de Interfaces Humano Tecnológica: Produto, Informações Ambientes Construídos e Transporte

$16^{\circ}$ USIHC - Congresso Internacional de Ergonomia e Usabilidade de Interfaces Humano Computador

CINAHPA | 2017 - Congresso Internacional de Ambientes Hipermídia para Aprendizagem.

\section{Introdução}

Órteses são tecnologias assistivas (TA) empregadas no tratamento de diferentes disfunções musculoesqueléticas. Segundo a OMS [2001 apud COOK E POLGAR, 2015 p.2] TA é qualquer produto, instrumento, equipamento ou tecnologia adaptado ou especialmente projetado para melhorar o funcionamento de uma pessoa com deficiência.

Edwards; Fenwick [2001, p. 237] definem que as órteses atuam como um sistema de forças (geralmente de três pontos) que agem sobre segmentos ou uma combinação de segmentos (membros superiores e inferiores) do corpo humano com debilidades ou deformidades, visando aprimorar as funções dos segmentos e a realização de tarefas pelo indivíduo. Entre as principais funções das órteses, por meio da imobilização, estão: manutenção do alinhamento, proteção, estabilização de articulações, aumento da amplitude de movimento, correção de deformidades, redução da dor e prevenção de lesões nestes segmentos. São utilizadas, sobretudo, em debilidades articulares e motoras, anomalias congênitas ou adquiridas. A configuração de uma órtese deve ser aquela capaz de adaptar-se à geometria corporal, à antropometria e à biomecânica de indivíduos específicos. Portanto, a qualidade das órteses está diretamente ligada às técnicas, equipamentos e materiais utilizados no desenvolvimento e fabricação das mesmas.

As órteses devem ser consideradas por especialistas em tratamentos médicos a partir da análise anatômica - e de possíveis deformidades muscular e da amplitude de movimento dos pacientes, tendo em vista a definição do tipo de órtese a ser adotada, bem como, seus materiais e as considerações estéticas (material, cores, acabamentos) da órtese [EDWARDS; FENWICK, 2001, p. 240]. Segundo os autores, visando o sucesso da inserção da órtese em tratamentos, o terapeuta ocupacional fabrica órteses específicas para cada paciente a partir de termoplásticos, ou faz adaptações de órteses comerciais conforme a anatomia do paciente. Em ambos os casos precisa considerar o diagnóstico médico. Ou seja, ocorre a produção customizada desses dispositivos considerando-se a adaptação do produto ao corpo do indivíduo e às atividades a serem realizadas com a utilização da órtese.

Na fabricação de órteses customizadas utiliza-se tradicionalmente o processo baseado na retirada de moldes dos segmentos do corpo nos quais se pretende intervir. Este método é satisfatório, contudo, a coleta antropométrica pode ser invasiva e desconfortável para o paciente, ao exigir posições específicas, demasiado tempo e quantidade de material. Problemas hoje evitados em clínicas mais modernas, que utilizam novas tecnologias como o scanner 3D e softwares CAD [JIN ET AL ,2015] O processo digital inicia-se com o escaneamento do membro do paciente. O modelo escaneado é processado em software CAD para o desenvolvimento da órtese em ambiente digital. Posteriormente é fabricada por manufatura aditiva (por exemplo, a impressão 3D) e então avaliada em relação ao usuário.

Nesse panorama, o estudo aqui apresentado visa realizar uma investigação com os objetivos de identificar: quais os processos utilizados por diferentes especialistas no desenvolvimento e fabricação de órteses customizadas, as principais etapas dos processos, as discrepância e paridades entre os processos, as tecnologias e processos produtivos utilizados para a fabricação das órteses.

\section{Método}

Para a busca dos estudos realizou-se em julho de 2015 uma revisão bibliográfica sistematizada nas bases de dados cientificos: Scopus, Science Direct, Periódicos Capes e Pub Med. Foram utilizados os strings de busca "cutomizated", "orthosis", "customization", nos pares: "cutomizated orthosis" e "cutomization orthosis". Os critérios adotados na seleção dos estudos foram: 1 - Leitura do título; 2 - Leitura do abstract e verificação da presença de desenvolvimento de órtese customizada; 3 - Leitura completa do artigo a fim de verificar a descrição de todas as etapas utilizadas no processo de customização. Resultados repetidos entre os bancos de dados foram eliminados, bem como, diferentes artigos de
Realização:

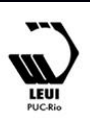




\section{$16^{\circ}$ \\ ERGODESIGN USIHC CINAHPA}

$16^{\circ}$ Ergodesign - Congresso Internacional de Ergonomia e Usabilidade de Interfaces Humano Tecnológica: Produto, Informações Ambientes Construídos e Transporte

$16^{\circ}$ USIHC - Congresso Internacional de Ergonomia e Usabilidade de Interfaces Humano Computador

CINAHPA | 2017 - Congresso Internacional de Ambientes Hipermídia para Aprendizagem. mesmos autores, que descreveram/utilizaram os mesmos processos. No total foram selecionados 05 estudos que estavam em conformidade com os requisitos citados.
As análises foram elaboradas e tabeladas visando à identificação das etapas do processo de desenvolvimento de órteses customizadas, apresentadas na Tabela 1 .

\section{Resultados}

\subsection{Estudos Analisados}

\begin{tabular}{|c|c|c|c|}
\hline Autores/ano & Título & $\begin{array}{l}\text { Tipo de órtese e } \\
\mathrm{N}^{\circ} \text { de sujeitos }\end{array}$ & Etapas percorridas no desenvolvimento \\
\hline $\begin{array}{l}\text { Schrank; } \\
\text { Stanhope } \\
{[2011]}\end{array}$ & $\begin{array}{l}\text { Dimensional accuracy } \\
\text { of ankle-foot orthoses } \\
\text { constructed by rapid } \\
\text { customization and } \\
\text { manufacturing } \\
\text { framework }\end{array}$ & $\begin{array}{l}\text { Passive-dynamic } \\
\text { Ankle-foot } \\
\text { Orthosis } \\
\mathrm{n}=2\end{array}$ & $\begin{array}{l}\text { - Fase de caracterização do sujeito: caracterizar o ajuste; caracterizar a função; } \\
\text { - Alinhamento virtual ortopédico dos pontos de referência: criar segmentos } \\
\text { anatômicos; registrar os pontos de referência; alinhar articulação virtual; } \\
\text { - Customização do modelo CAD: dimensionar modelo em CAD; selecionar os } \\
\text { parâmetros de design; ajustar as características funcionais; } \\
\text { - Fabricação: utilizando Selective Laser Sintering (SLS); } \\
\text { - Avaliação: Avaliação da precisão dimensional e avaliar a rigidez da órtese. }\end{array}$ \\
\hline $\begin{array}{l}\text { Crabtree; } \\
\text { Dhoki; } \\
\text { Newman; } \\
\text { Ansell [2009] }\end{array}$ & $\begin{array}{l}\text { Manufacturing } \\
\text { methodology } \\
\text { for personalized } \\
\text { symptom specific } \\
\text { sports insoles }\end{array}$ & $\begin{array}{l}\text { Palmilha } \\
\text { esportiva, } \\
\text { adequada às } \\
\text { necessidades de } \\
\text { esporte específico. } \\
\mathrm{n} / \mathrm{e}^{*}\end{array}$ & $\begin{array}{l}\text { - Análise biomecânica do paciente; } \\
\text { - Prescrição do paciente; } \\
\text { - Design em CAD e escolha de materiais; } \\
\text { - Método de fabricação; } \\
\text { - Entrega da Palmilha com avaliação da mesma, pelo paciente.. }\end{array}$ \\
\hline $\begin{array}{l}\text { Fortin et al } \\
\text { [2007] }\end{array}$ & $\begin{array}{l}\text { A } 3 D \text { visualization tool } \\
\text { for the design and } \\
\text { customization of spinal } \\
\text { braces }\end{array}$ & $\begin{array}{l}\text { Colete para } \\
\text { escoliose. } \\
n=2\end{array}$ & $\begin{array}{l}3 \text { Macro etapas: } \\
\text { - } 1^{\text {a }} \text { visita: Aquisição da anatomia do paciente: aquisição da anatomia interna e } \\
\text { externa do paciente para que seja feito o design da órtese; } \\
\text { - Processamento dos dados e design do colete; } \\
\text { - } 2^{\text {a }} \text { visita: Posicionamento e ajuste do colete. }\end{array}$ \\
\hline $\begin{array}{l}\text { Alam; } \\
\text { Choudhury; } \\
\text { Bin Mamat } \\
{[2015]}\end{array}$ & $\begin{array}{l}\text { Computer aided design } \\
\text { and fabrication } \\
\text { of a custom articulated } \\
\text { ankle foot orthosis }\end{array}$ & $\begin{array}{l}\text { Articulated Ankle } \\
\text { Foot Orthosis. } \\
\mathrm{n}=1\end{array}$ & $\begin{array}{l}\text { - Aquisição dos dados antropométricos do paciente; } \\
\text { - Design da órtese; } \\
\text { - Fabricação da órtese; } \\
\text { - Validação da órtese através da análise da marcha do paciente. }\end{array}$ \\
\hline $\begin{array}{l}\text { Kaiser et al } \\
\text { [2014] }\end{array}$ & $\begin{array}{l}\text { Case study: mass } \\
\text { customization of } \\
\text { individualized } \\
\text { orthotics - the } \\
\text { fashion-able virtual } \\
\text { development and } \\
\text { production framework }\end{array}$ & $\begin{array}{l}\text { Colete para } \\
\text { tratamento de } \\
\text { escoliose. } \\
\mathrm{n}=2\end{array}$ & $\begin{array}{l}\text { - Requisitos do usuário: coleta destes requisitos fornecidos por médicos; } \\
\text { - Configuração: informação gera uma órtese específica, que terá } \\
\text { características especificas de acordo com a doença tratada; } \\
\text { - Geração do Protótipo virtual: desenvolvimento da órteses; colocação virtual } \\
\text { da órtese. Adição de informação de funcionalidade específica; } \\
\text { - Produção Customizada; } \\
\text { - Entrega. }\end{array}$ \\
\hline
\end{tabular}

Tabela 1: Artigos selecionados para estudo, analisados quanto às etapas dos processos adotadas pelos autores e o número de sujeitos participantes do estudo. ${ }^{*} \mathrm{n} / \mathrm{e}=$ dados não especificados pelos autores.

\subsubsection{Schrank; Stanhope [2011]}

O processo de customização apresentado pelos autores foca na automatização. É apresentado por meio do desenvolvimento de uma órtese para tornozelo e pé (AFO) passivo-dinâmica.

O processo de customização e produção é composto de cinco macro fases: Fase 1.

Caracterização do sujeito: São escaneados pontos de referencia $(n=45)$, que geram em software o formato do membro inferior do sujeito. Fase 2. Alinhamento virtual ortopédico dos pontos de referência: em um software, os dados capturados do membro são alinhados com um sistema de coordenadas de pontos de referência de um modelo 


\section{$16^{\circ}$ \\ ERGODESIGN USIHC CINAHPA}

de membro inferior preexistente. Fase 3. Customização do Modelo CAD: nessa etapa a órtese em si é desenvolvida a partir da união dos pontos de referência já alinhados, previamente escaneados, e com parâmetros de design preestabelecidos. Fase 4. Fabricação: A órtese é produzida em SLS. Fase 5. Avaliação: é realizada uma avaliação em relação à adequação antropométrica da órtese.

\subsubsection{Crabtree et al [2009]}

Os autores descrevem o método de desenvolvimento da Symptom-Specific Sports (3S) Insole. O processo leva em consideração além dos aspectos biomecânicos do pé do indivíduo, as forças que o pé sofre e exerce durante a prática esportiva, que variam de acordo com o esporte.

Primeiramente é realizada a avaliação dos requisitos biomecânicos: A partir de um protocolo validado, são avaliados os requisitos do pé do paciente (através do escaneamento da geometria do pé e mapeamento das áreas de pressão), do esporte praticado e do calçado utilizado na prática do esporte. Os dados gerados são cruzados com um banco de dados de materiais, que busca por materiais com propriedades mecânicas mais adequadas ao esporte praticado, pois a escolha de material também influenciará diretamente no design da órtese. Em seguida, o design da órtese é desenvolvido em software CAD, a partir do modelo gerado da geometria do pé alinhado com a geometria da parte interna do calçado a ser utilizado pelo paciente. Para a fabricação da órtese, os autores sugerem a utilização dos métodos de laminação de fibra de carbono e usinagem criogênica.

\subsubsection{Fortin et al [2007]}

O objetivo dos autores foi o de desenvolver um software que auxilie o profissional da saúde na elaboração de faixas de compressão para a coluna. O software permite a visualização da geometria do segmento do corpo do paciente em três dimensões (3D), visando a substituição do uso tradicional do raio $\mathrm{X}$. O processo de desenvolvimento é um protocolo composto de três macro fases: 1 . A visita $16^{\circ}$ Ergodesign - Congresso Internacional de Ergonomia e Usabilidade de Interfaces Humano Tecnológica: Produto, Informações Ambientes Construídos e Transporte

$16^{\circ}$ USIHC - Congresso Internacional de Ergonomia e Usabilidade de Interfaces Humano Computador

CINAHPA | 2017 - Congresso Internacional de Ambientes Hipermídia para Aprendizagem. inicial do paciente na clínica de escoliose para a prescrição do colete; 2. Fabricação do colete; 3 . Segunda visita do paciente para avaliação e ajuste do colete. A duração do processo é de um mês.

Na Fase 1: Um Pressure Mat (uma pequena esteira com sensores de pressão) é fixado no tronco do paciente que está com 12 marcações (para facilitar o reposicionamento na segunda visita). A seguir, sem o Pressure Mat é feita a aquisição dos dados referentes à geometria do tronco do paciente, com 4 digitalizadores ópticos 3D, com o paciente localizado no centro de uma instalação de digitalizadores. Na Fase 2: São processadas as imagens digitalizadas do paciente, gerando em um software específico, um modelo da parte interna e externa do troco do paciente. Neste software o especialista inicia o desenvolvimento da órtese, com a possibilidade de determinar as regiões nas quais deseja inserir áreas de pressão e áreas de alívio na órtese. Na Fase 3: Ocorre a segunda visita e reposiciona-se no paciente o Pressure Mat e veste-se o colete prescrito. $\mathrm{O}$ especialista tem condições de avaliar as pressões exercidas pelo colete para determinar se condiz com a prescrição.

\subsubsection{Alam et al [2015]}

Os autores descrevem o processo utilizado para o desenvolvimento e fabricação de uma órtese customizada para tornozelo e pé. O processo inicia-se por meio da obtenção dos dados antropométricos do membro inferior de um indivíduo saudável, com auxílio da tomografia computadorizada, que gera um modelo virtual em duas camadas ou dois modelos virtuais: um modelo dos tecidos externos do paciente e um modelo esquelético. Em seguida, o modelo virtual ajusta-se segundo modelos de referência de posicionamento articular preexistentes chamados VAKHUM (Virtual Animation Of The Kinematics Of The Human For Industrial, Educational And Research Purposes). Após essa etapa, o modelo virtual serve de referência para o dimensionamento da órtese em software CAD, posteriormente fabricado em chapas de alumínio. Finalmente, realiza-se a análise da marcha do paciente com a órtese customizada, no intuito de compará-la com órteses pré-fabricadas. 


\section{$16^{\circ}$ \\ ERGODESIGN USIHC CINAHPA}

$16^{\circ}$ Ergodesign - Congresso Internacional de Ergonomia e Usabilidade de Interfaces Humano Tecnológica: Produto, Informações Ambientes Construídos e Transporte

$16^{\circ}$ USIHC - Congresso Internacional de Ergonomia e Usabilidade de Interfaces Humano Computador

CINAHPA | 2017 - Congresso Internacional de Ambientes Hipermídia para Aprendizagem.

\subsubsection{Kaiser et al [2014]}

$\mathrm{O}$ artigo apresenta o framework desenvolvido pelo projeto FASHION-ABLE, financiado pela Comissão Europeia, que objetiva fornecer a pequenas e médias empresas europeias, tecnologias inovadoras e sustentáveis. Estas tecnologias devem servir para produtos vestíveis a pessoas com necessidades especiais. O framework divide-se em duas etapas de trabalho: a aquisição de dados, e os processos de desenvolvimento da órtese.

As etapas do framework descritas pelos autores são: 1. Requisitos: os requisitos específicos do paciente são providos antecipadamente por um médico; 2. Configuração: informações são coletadas para a configuração da órtese para o paciente, ou seja, a definição do uso de materiais, de mecanismos de fechamento, reforços, áreas de compressão e a utilização funcional de tecidos; 3 . Geração do protótipo virtual: uma configuração especifica é gerada para garantir o seu ajuste. Ainda nessa etapa, realiza-se a simulação da colocação da órtese em um manequim virtual acomodado no corpo do paciente, adicionando-se áreas funcionais dos materiais aplicados, de acordo com a doença do paciente; 4. Produção Customizada; 5. Entrega.

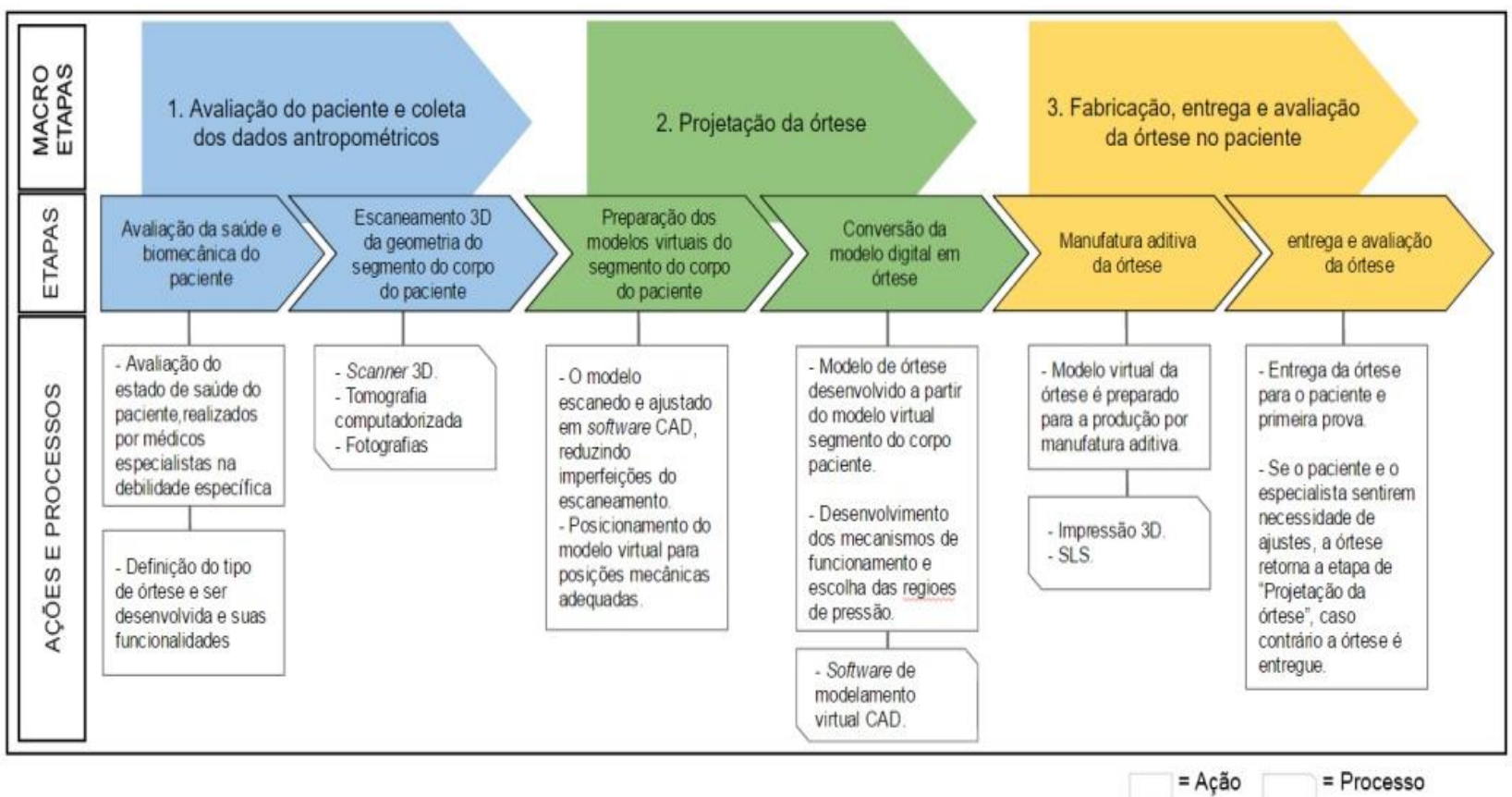

Figura 1. Processo abrangente para o desenvolvimento de órtese customizada baseado nos estudos de Schrank; Stanhope [2011], Crabtree et al [2009], Fortin et al [2007], Alam et al [2015], Kaiser et al [2014].

\section{Discussões}

A aquisição de dados antropométricos fidedignos, tanto aqueles relativos à fisionomia como ao sistema musculoesquelético do paciente, mostrouse o principal fator para a customização nos processos analisados nesse trabalho. Da mesma forma, aos aspectos biomecânicos relacionados ao uso das órteses. Portanto, a customização existe nos processos analisados somente quando há adequação da órtese à geometria do segmento do corpo do paciente, em conjunto com avaliações médicas. Ademais, percebe-se que nas etapas de obtenção dos dados antropométricos, todos os estudos fizeram o uso de algum tipo de tecnologia não invasivo como scanners $3 \mathrm{D}$ e tomografia, que geram dados antropométricos precisos e reduzem o tempo consumido nessa etapa. São nas etapas de aquisição de dados antropométricos e na etapa de projetação que se observam as principais diferenças entre os processos analisados e aqueles
Realização:
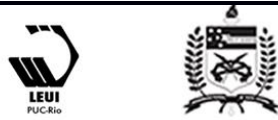


\section{$16^{\circ}$ \\ ERGODESIGN USIHC CINAHPA}

tradicionais como apresentados por Jin et al [2015]. Nessas etapas, as tecnologias digitais de obtenção de dados antropométricos associam-se a softwares CAD, como pode ser observado em todos os estudos. Essa combinação de tecnologias proporciona a execução de uma etapa de projetação com liberdade no desenvolvimento de soluções, pois o projeto virtual pode ser modificado e reajustado inúmeras vezes sem consumir os materiais de fabricação ou exigir novas medições. A etapa de projetação torna-se mais precisa no que tange à adequação antropométrica e aos estudos mecânicos de resistência dos materiais e aos pontos de pressão aplicados ao corpo do paciente. O software CAD permite também a reprodutibilidade do projeto, pois uma órtese pode ser fabricada diversas vezes utilizando-se o mesmo arquivo CAD.

\section{Conclusão}

$\mathrm{Na}$ análise dos processos de desenvolvimento autorais estudados, observaram-se macro etapas em comum, podendo-se dizer que os processos de customização de órteses consistem em três macro etapas: 1. A avaliação do paciente e coleta de dados antropométricos 2. Projetação da órtese. 3.

Fabricação, entrega e avaliação da órtese no paciente. A sumarização do processo refere-se aos estudos analisados e podem não representar o que é amplamente utilizado por especialistas.

Os processos analisados não apresentam discrepâncias em relação àqueles tradicionais, no que diz respeito às suas macro etapas, porém, há um foco claro na automatização, redução de etapas e no tempo consumido nos processos. O enfoque dos processos de customização está, portanto, na obtenção de dados precisos do indivíduo, tanto relativos à saúde, à antropometria, à liberdade criativa e à fácil produção e avaliação das órteses desenvolvidas.

\section{BIBLIOGRAFIA}

ALAM, M. et al. Computer aided design and fabrication of a custom articulated ankle foot orthosis. Journal of Mechanics in Medicine and $16^{\circ}$ Ergodesign - Congresso Internacional de Ergonomia e Usabilidade de Interfaces Humano Tecnológica: Produto, Informações Ambientes Construídos e Transporte

$16^{\circ}$ USIHC - Congresso Internacional de Ergonomia e Usabilidade de Interfaces Humano Computador

CINAHPA | 2017 - Congresso Internacional de Ambientes Hipermídia para Aprendizagem.

Biology, v. 15, n. 04, p. 1550058, 2015.

CRABTREE, P. et al. Manufacturing methodology for personalised symptom-specific sports insoles.

Robotics and Computer-Integrated

Manufacturing, v. 25, n. 6, p. 972-979, 2009.

COOK, A.M.; POLGAR, J.M. Assistive technologies: Principles and practice. Elsevier Health Sciences, 2015.

FORTIN, D. et al. A 3D visualization tool for the design and customization of spinal braces. Computerized Medical Imaging and Graphics, v. 31, n. 8, p. 614-624, 2007.

EDWARDS, J.; FENWICK, L. In: OLSON, D. A.; DERUYTER, F. Clinician's guide to assistive technology. Mosby Inc, 2001. cap 14, p.237-249.

JIN, Y. et al. Additive manufacturing of custom orthoses and prostheses-A review. Procedia CIRP, v. 36, p. 199-204, 2015.

KAISER, C.et al. Case Study: Mass Customisation of Individualized Orthotics-The FASHION-ABLE Virtual Development and Production Framework. Procedia CIRP, v. 21, p. 105-110, 2014.

SCHRANK, E. S. Dimensional accuracy of anklefoot orthoses constructed by rapid customization and manufacturing framework. Journal of rehabilitation research and development, v. 48, n. 1, p. 31, 2011.

\section{Agradecimentos}

Gostaríamos de agradecer à Universidade do Estado de Santa Catarina (UDESC), ao Programa de Pós Graduação em Design - PPGDesign (UDESC) e à CAPES pelo apoio durante a realização da pesquisa. 